\title{
The reporting of a Bacillus anthracis B-clade strain in South Africa after more than 20 years
}

\author{
K. E. Lekota ${ }^{1,5^{*}}$, A. Hassim ${ }^{1}$, P. Rogers ${ }^{2}$, E. H. Dekker ${ }^{3}$, R. Last ${ }^{4}$, L. de Klerk-Lorist ${ }^{3}$ and H. van Heerden ${ }^{1}$
}

\begin{abstract}
Objectives: Anthrax is a disease with an age old history in Africa caused by the Gram-positive endospore forming soil bacterium Bacillus anthracis. Epizootics of wild ungulates occur annually in the enzootic region of Pafuri, Kruger National Park (KNP) in the Limpopo Province of South Africa. Rigorous routine surveillance and diagnostics in KNP, has not revealed these rare isolates since the 1990s, despite unabated annual outbreaks. In 2011 a cheetah was diagnosed as anthrax positive from a private game reserve in Limpopo Province and reported to State Veterinary Services for further investigation. Isolation, molecular diagnostics, whole genome sequencing and comparative genomics were carried out for B. anthracis KC2011.

Results: Bacteriological and molecular diagnostics confirmed the isolate as B. anthracis. Subsequent typing and whole genome single nucleotide polymorphisms analysis indicated it clustered alongside B. anthracis SA A0091 in the B.Br.010 SNP branch. Unlike B. anthracis KrugerB strain, KC2011 strain has unique SNPs and represents a new branch in the B-clade. The isolation and genotypic characterisation of KC2011 demonstrates a gap in the reporting of anthrax outbreaks in the greater Limpopo province area. The identification of vulnerable and susceptible cheetah mortalities due to this strain has implications for conservation measures and disease control.
\end{abstract}

Keywords: Bacillus anthracis, Whole genome sequencing (WGS), Single nucleotide polymorphisms (SNPs)

\section{Introduction}

Anthrax is a zoonotic disease with an age old history in Africa [1], causing acute mortalities. It primarily affects ungulates with episodic spill over into humans and carnivores [2,3]. The causative agent of this disease is the Gram positive, endospore forming, exotoxin producing soil bacterium Bacillus anthracis [4,5]. Sporulation is triggered by nutrient scarcity, the presence of bicarbonate and oxygen exposure $[6,7]$. The virulence factors of this bacterium are on the two plasmids, pXO1 (181 kilobases) and pXO2 (94 kilobases), encoding the toxin and capsule genes respectively $[8,9]$. The toxin complex is composed of three components namely, lethal toxin (LF) made up of the protective (PA) and lethal factor (EF) $[10,11]$. The

\footnotetext{
*Correspondence: Lekotae@gmail.com

${ }^{1}$ Department of Veterinary Tropical Diseases, Faculty of Veterinary Science, University of Pretoria, Onderstepoort, South Africa

Full list of author information is available at the end of the article
}

capsule consists of a five gene operon (capBCADE) that produces a poly-gamma-D-glutamic acid (PGA) on the bacterial cell membrane that protects the vegetative cells from phagocytosis $[12,13]$.

Anthrax was widespread across southern Africa in the early 20th Century, until the development and South African state implementation of the Sterne vaccine in 1937 [14, 15]. While livestock cases have since been infrequent due to vaccination practices; outbreaks are still common amongst wildlife in endemic regions of South Africa and game reserves across the continent $[2,16]$. Limpopo province covers $125,754 \mathrm{~km}^{2}$ bordering Botswana and Zimbabwe to the north. The northern half of the Kruger National Park (KNP) makes up the entire eastern border of the Limpopo Province bordering Mozambique. The anthrax endemic region in KNP falls between the Limpopo and Luvhuvhu rivers [19]. The distribution of outbreaks and the animal species affected by anthrax have been described extensively in KNP in the 
last 35 years [17-20]. The genetic population structure of B. anthracis consists of the global A, B and C clades [21]. The B clade isolates identified in South Africa were confined to the northern tip of KNP in Limpopo Province due to it being an ideal soil environment for spore persistence [17]. The A clade isolates, in contrast, have a wide distribution, predominating and prevailing over the rarer B clade isolates during outbreaks since the late 1980s.

Host species differ in their susceptibility to $B$. anthracis. Wild ungulates are more susceptible to the disease than carnivores $[2,3]$. Even amongst herbivores, roan antelope (Hippotragus equinus) are far more susceptible to anthrax than other antelope species [20]. Similarly, cheetahs (Acinonyx jubatus) are more susceptible to the toxins produced by the bacterium than other carnivorous felids and canids [22-24]. It is hypothesised that this could be due to the immune response dictated by the monomorphic genetic structure of southern African cheetahs [25] or by the feeding habits of free roaming cheetah [22]. In either event, it is of importance to cheetah conservation practices since these felids are considered vulnerable (bordering on endangered) [26].

This status has led to more stringent investigations of cheetah mortalities in both captive and free roaming animals. In 2011 a cheetah mortality from a private game reserve in Limpopo Province was investigated by the local veterinarian. The cheetah was observed to have substantial facial oedema with unclotted blood around the kidneys (Fig. 1A, B). Biological samples from the cheetah were submitted for bacteriologic diagnostics (Fig. 1C, $D)$. Colony morphology indicative of $B$. anthracis then confirmed the initial diagnosis (Fig. 1D). The isolate was
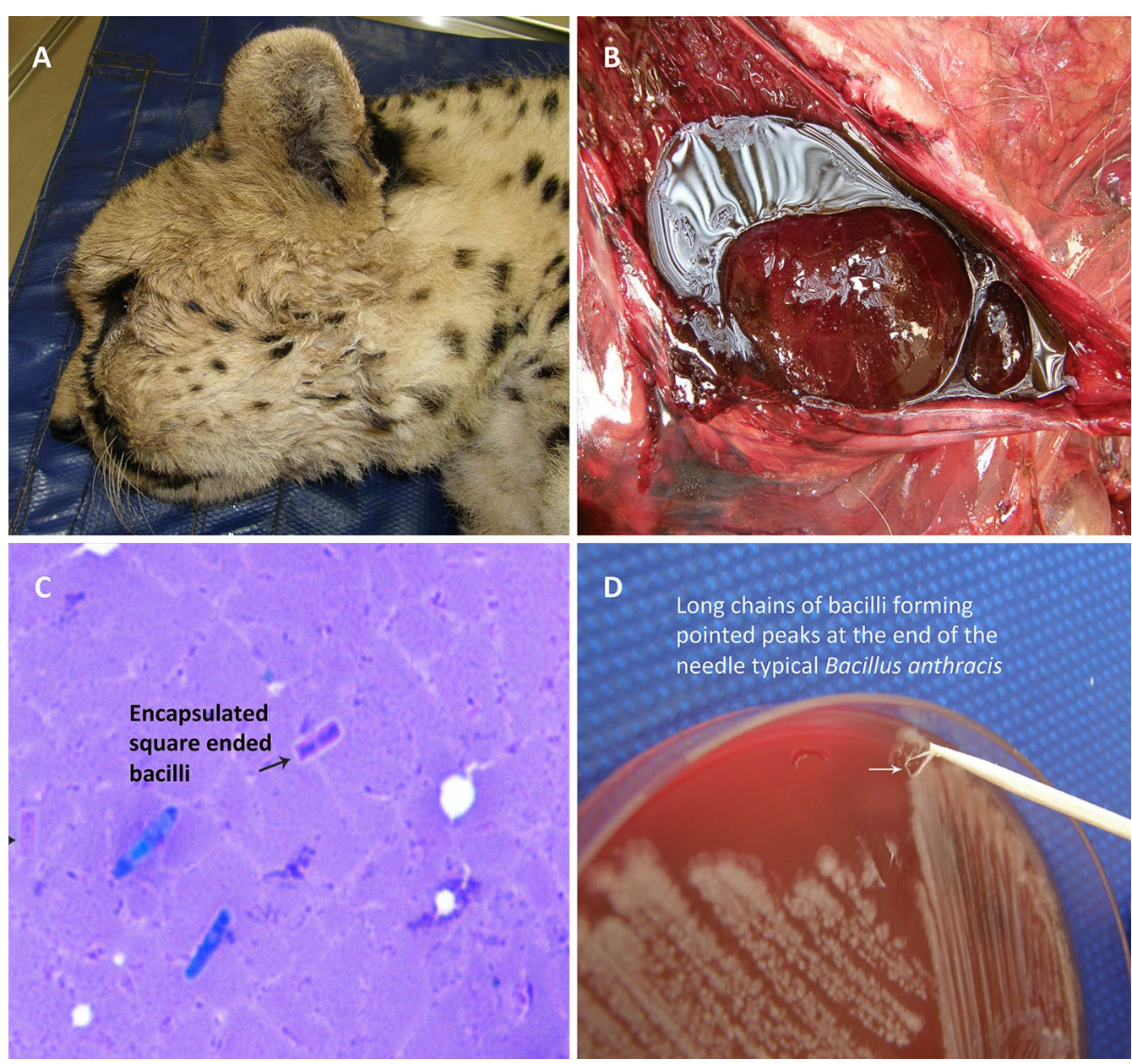

Fig. 1 A Cheetah (Acinonyx jubatus) demonstrating facial oedema in reaction to Bacillus anthracis toxins. B Dark un-clotted blood surrounding the kidney of a cheetah indicating infection by $B$. anthracis.C Impression smear of oedematous fluid from cheetah with typical square ended $B$. anthracis bacilli (purple bacilli indicated by the arrow). D Bacteriologic diagnostics on 5\% sheep blood agar demonstrating typical B. anthracis colony morphology and structure 
then submitted by Skukuza State Veterinary Services for further analysis on behest of the Anthrax Advisory Committee.

\section{Main text \\ Methods \\ Isolation and identification}

A Giemsa smear stain from the oedema fluid was performed for identification of $B$. anthracis. Pure culture isolation of KC2011 isolate was obtained by culturing biological samples directly onto 5\% sheep blood agar after $24 \mathrm{~h}$ incubation at $37{ }^{\circ} \mathrm{C}$ characteristics including colony and cell morphology, gamma-phage and penicillin sensitivity were evaluated as described in OIE [3]. Capsule formation was observed by visualization using light microscopy of Giemsa stained fluid and colonies.

\section{DNA extractions and $q P C R$}

Genomic extraction of B. anthracis KC2011 was carried out using a DNA Blood Mini Kit (Qiagen, Germany) according to manufacturer's instructions. The DNA was quantified using Qubit ${ }^{\mathrm{TM}}$ fluorometric quantitation (Invit$\operatorname{rogen}^{\mathrm{TM}}$, USA). Quality of the extracted DNA was visualized on $0.8 \%$ agarose gel electrophoresis. The diagnostic qPCR for B. anthracis was performed with $2.5 \mu \mathrm{L}$ DNA in $1 \times$ FastStart $^{\mathrm{TM}}$ Taq DNA Polymerase mastermix (Roche ${ }^{\circledR}$, Germany) and $0.5 \mu \mathrm{M}$ of each primer small acid soluble proteins (SASP), Bacillus anthracis protective antigen (BAPA), and capsule region ( $\operatorname{cap} \mathrm{C})$ along with $0.2 \mu \mathrm{M}$ of probe for each chromosomal and plasmid target pairs with fluorescein on the one and LCRed640 on the other (Tib MolBiol GmbH, Germany) in a final volume of $20 \mu \mathrm{L}$ as described in Turnbull [3]. The PCR conditions on a LightCycler $^{\mathrm{TM}}$ Nano (Roche ${ }^{\circledR}$, Germany) were used as described in OIE $[3,27]$.

\section{Melt-MAMA assay}

The PCR single nucleotide polymorphism (SNP) assay was performed for the oligonucleotide markers $\mathrm{A} / \mathrm{B}$. Br.001, B.Br.003, B.Br.004, B.Br.002, B.Br.001, A.Br.007 and A.Br.002 as described in Birdsell et al. [28]. The reaction included $2.5 \mu \mathrm{L}$ DNA diluted in $1 \times$ FastStart DNA Green Master (Roche ${ }^{\circledR}$, Germany) with an ancestral forward and a derived forward SNP target primer (GCclamp: no-GC-clamp) and a common reverse primer with a starting concentration of $0.2 \mu \mathrm{M}$ depending on the ratio indicated which allowed for separation of melt peaks by at least $5{ }^{\circ} \mathrm{C}$. Thermocycling parameters on the LightCycler ${ }^{\mathrm{TM}}$ Nano (Roche ${ }^{\circledR}$, Germany) were conducted as described in Birdsell et al. [28].

\section{Whole genome sequencing and Bioinformatics analysis}

Sequence libraries of the DNA isolate was generated using the Nextera DNA Sample Prep Kit (Illumina, USA) protocol. Sequence reads of paired end library was performed on a HiSeq 2500 sequencer (Illumina, USA). Quality of the genome sequenced reads were assessed using FastQC software 0:10.1 [29]. Trimmomatic [30] was used to remove the ambiguous nucleotide reads. De novo assembly of the $B$. anthracis KC2011 was carried out using the CLC Genomics Workbench version 7.5 (CLC, Denmark). The assembled contigs were aligned with BLASTn [31] using B. anthracis Ames ancestor (GenBank: AE017334.2, AE017336.2, and AE017336.2) as a reference. Mauve tool [32] was used to align and order the assembled contigs using $B$. anthracis Ames ancestor as a reference. The genome was annotated using PGAAP at NCBI [33].

The trimmed reads of $B$. anthracis KC2011 were aligned to $B$. anthracis Ames ancestor using the BurrowsWheeler Aligner (BWA) [34]. SAMtools [35] was used to sort and index the aligned sequenced reads. Unified genotyper in GATK [36] was used to call for SNPs. In order to construct WGS- SNPs tree, complete and draft genomes from different clades of $B$. anthracis available in NCBI Genbank (http://www.ncbi.nom.nih.gov) were included in this study (Additional file 1: Table S1). SNPs positioning sets were deducted from the aligned genomes of $B$. anthracis Ames ancestor using molecular evolutionary genetics analysis (MEGA) 7 [37]. SNPs with informative sites (core SNPs) in all genome sequences were used for the phylogenetic tree construction using MEGA 7 tool [37].

\section{Genbank submission}

The genome sequence of B. anthracis KC2011 was deposited in the Genbank genome database under the accession number: NJGK00000000.

\section{Results}

\section{Isolation and identification}

A Giemsa stained impression smear from the oedema fluid revealed encapsulated, square ended bacilli pathognomonic for B. anthracis (Fig. 1C). The microbiological characteristics confirmed the bacterium to be $B$. anthracis on blood smear with typical $B$. anthracis colony morphology and structure on 5\% sheep blood agar [38] (Fig. 1D).

\section{qPCR and Melt-MAMA analysis}

The B. anthracis KC2011 isolate was confirmed positive for the presence of $B$. anthracis BAPA, SASP and capC. B. anthracis $\mathrm{KC} 2011$ was further typed using 
Melt-MAMA and amplified the derived markers for A/B.Br.001, B.Br.003 and B.Br.002, whilst amplifying the B.Br.001 ancestral marker. This SNP profile indicated KC2011 to be a B clade (B.Br.001/002), but not the rare KrugerB sub-clade.

\section{Genome assembly and phylogeny}

De novo assembly was carried out which resulted in 45 contigs (Table 1). The total genome contigs contributed to a $5.42 \mathrm{MB}$, with a GC content of 35\%. A total of 6047 coding sequences (CDSs) was determined in the KC2011 strain (Table 1). Comparative genome alignment of the $B$. anthracis KC2011 genome with Ames ancestor revealed no evidence of novel genes (Additional file 3: Fig. S1). The genome coverage of B. anthracis KC2011 was 99\% covered to $B$. anthracis Ames ancestor (Additional file 1: Table S1; Additional file 2: Table S2). High copy number of pXO1 was observed represented by 725 coverage $(725 \times)$.

A high resolution tree of global $B$. anthracis strains was constructed to visualise the SNP grouping of the $B$. anthracis KC2011 (Fig. 2). This yielded 3247 parsimony informative SNPs that were used to construct the phylogram. The B. anthracis KC2011 grouped in the B-clade separate from the KrugerB strain. The closest related branch was SA B. anthracis A0091.

\section{Discussion}

Strain KC2011 is the first B. anthracis identified as a B-clade lineage bacterium in over 20 years in South Africa and represents a new subclade in the B-clade. About $90 \%$ of the anthrax outbreaks reported worldwide arise from the A-clade, while less than $10 \%$ have

Table 1 Genome features of the Bacillus anthracis KC2011

\begin{tabular}{ll}
\hline Features & $\begin{array}{l}\text { Bacillus anthracis } \\
\text { KC2011 genome }\end{array}$ \\
\hline Domain & Bacteria \\
Genome coverage (X) & 358 \\
Avg. length after trim & 118 \\
Genome size (bp) & 5421567 \\
Number of contigs & 45 \\
Maximum length & 500750 \\
Minimum length & 9000 \\
G+C content (\%) & 35.2 \\
Genes (total) & 6047 \\
CDS (coding) & 5964 \\
Genes (RNA) & 83 \\
Complete rRNAS & $4,1(5 \mathrm{~S}, 23 \mathrm{~S})$ \\
Number of tRNA & 73 \\
Number of ncRNAs & 5 \\
Pseudo genes (total) & 322 \\
\hline
\end{tabular}

been reported from the B-clade (KrugerB subclade) [21]. The majority of the $B$. anthracis strains isolated from the northern part of KNP from 1970 to 1981 grouped in the B-clade (KrugerB subclade) but this clade became rare since the 1990s [17]. Only one B strain reported between 1982 and 1997 [19] to the extent that it was no longer isolated from 1997 until this report in South Africa.

No significant anthrax outbreaks were noted outside the endemic regions of KNP (northern part of KNP) in 2011 according to state veterinary surveillance data. The isolate KC2011 was only identified because of the delicate conservation status of cheetah in Africa and because it was within the context of the broader Limpopo province area (i.e. outside KNP). Cheetahs are especially susceptible to the $B$. anthracis exotoxins, however, they do respond well to vaccination with the Sterne vaccine [25]. This study highlighted the importance of vaccinating cheetah, where logistically possible, in conservancies across Limpopo province.

The phylogenetic structure of $B$. anthracis determined in this study (Fig. 2) is similar to other reported WGSSNP trees [39-42]. Past B-clade (1965-1990) isolates from the State Veterinary archival collection have all clustered in the KrugerB lineage. WGS-SNP analysis defined KC2011 strain grouping as a distinctive sub-clade within southern Africa B.Br.010.

Although, distant in terms of number of SNPs, the isolate A0091 and KC2011 belong to a common albeit broader lineage. The isolate A0091 is recorded as being from South Africa circa 1939, but has no other information to link it to KNP or Limpopo Province. There is also a dearth of information from the countries (Zimbabwe and Mozambique) bordering the South African province across the Limpopo River. Further surveillance of Limpopo Province is required to reveal whether deposits of B-clade isolates are subsisting outside KNP or whether this was an incidental mortality due to circumstances outside the realm of current monitoring criteria. Surveillance in the non-endemic anthrax regions is difficult as there are private game reserves and rural livestock farmers bordering KNP. Moreover the rural subsistence farmers (predominantly cattle farmers) are less likely to report mortalities and/or vaccinate their livestock thus complicating disease monitoring and control. This report of the B. anthracis $\mathrm{KC} 2011$ isolated outside the endemic region highlights the necessity of disease surveillance in nonendemic regions.

\section{Conclusion}

The identification of $\mathrm{KC} 2011$ is significant in that mortalities linked to the B-clade have diminished over several decades and therefore was estimated to have disappeared from the local environment. This suggests a possible gap 


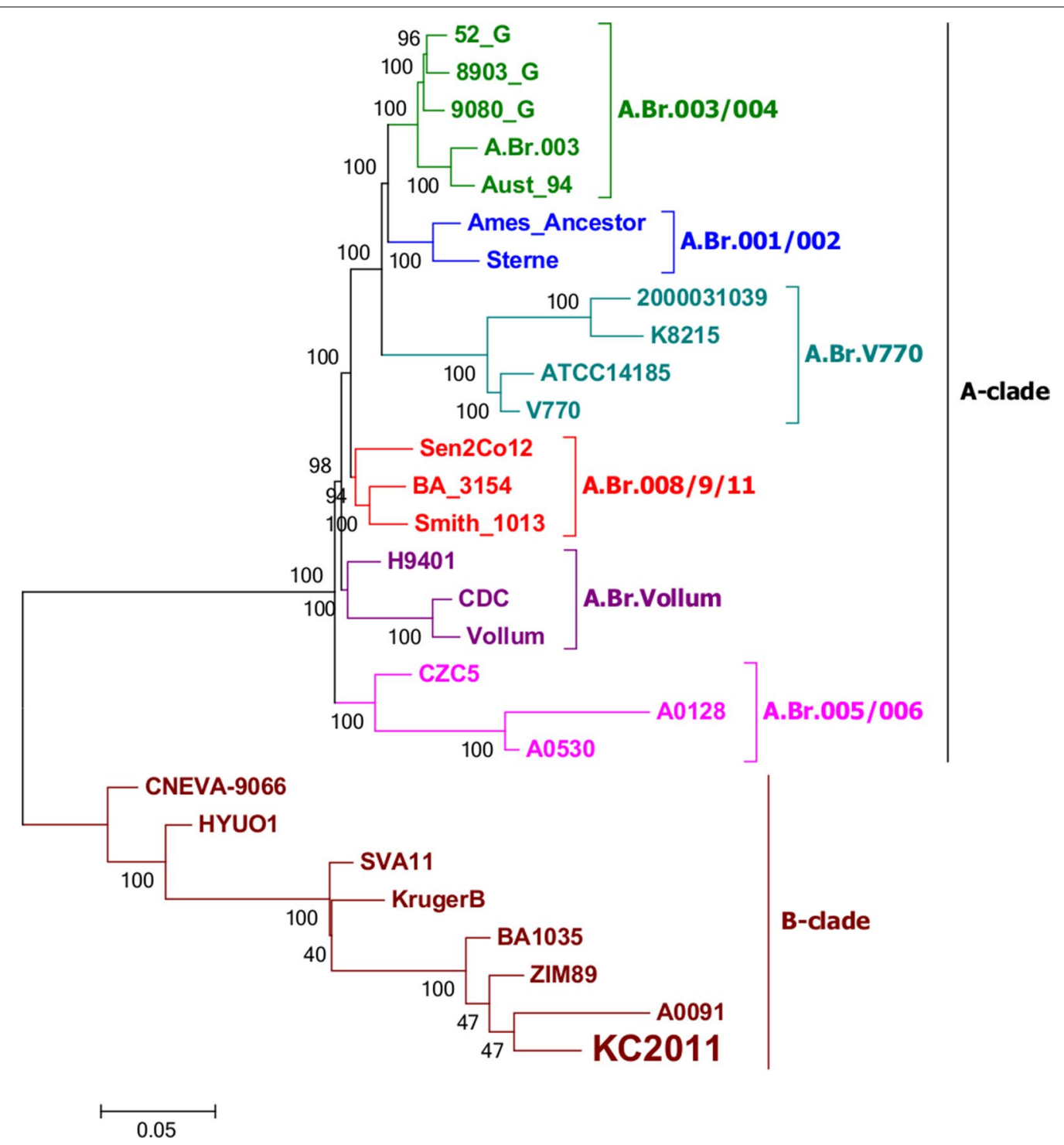

Fig. 2 Whole genome SNP phylogeny of the global Bacillus anthracis strains indicating the placement of KC2011 strain. Maximum likelihood was constructed using 3247 parsimony informative SNPs. The rare B. anthracis KC2011 (indicated with higher font) grouped in the B-clade separately from KrugerB strain

in surveillance of carcasses submitted for anthrax diagnostics in the Limpopo province especially in the nonendemic regions. It also has implications for the inclusion of the Sterne vaccine as part of the conservation measures to be included for cheetah in sanctuaries and private game reserves across the Limpopo Province in South Africa.

\section{Limitations}

The tree topology of the southern African strains was not well characterised due to the limited number of genomes available. Branch lengths vary according to inclusion of number and variety genomes in the data sets, as this explicates the informative SNPs used to construct the tree. Isolate $\mathrm{KC} 2011$ will thus be the basis 
for further typing of $B$. anthracis strains for southern Africa.

\section{Additional files}

Additional file 1: Table S1. Whole genomes of Bacillus anthracis retrieved from public database used in this study.

Additional file 2: Table S2. Genome alignment of the Bacillus anthracis KC2011 to the B. anthracis Ames ancestor reference.

Additional file 3: Fig. S1. Alignment of Bacillus anthracis Ames ancestor genome with B. anthracis KC2011. Each colour block indicates homologous regions of the genome sequences. White areas in colour blocks indicate possible nucleotide variation absence or presence within the compared genomes.

\section{Abbreviations}

PGA: poly-gamma-D-glutamic acid; LF: lethal toxin; PA: protective; EF: lethal factor; KNP: Kruger National Park; WGS: whole genome sequencing; SNP: single nucleotide polymorphism; Melt-MAMA: melting mismatch amplification mutation analysis; CDSs: coding sequences.

\section{Authors' contributions}

KEL and AH performed the molecular, as well as, in silico analysis and drafting of the manuscript. RL and PR identified the mortality and performed the pathology evaluation. LDKL and HED performed the bacterial and DNA isolations. HvH participated in the design of the study, drafting the manuscript, revising it critically and provided funding. All authors read and approved the final manuscript

\section{Author details}

${ }^{1}$ Department of Veterinary Tropical Diseases, Faculty of Veterinary Science, University of Pretoria, Onderstepoort, South Africa. ${ }^{2}$ Provet Wildlife Services, Raptors Safari Junction, Main Road, Hoedspruit, South Africa. ${ }^{3}$ State Veterinary Services, Department of Agriculture, Forestry and Fisheries, Skukuza, South Africa. ${ }^{4}$ Vetdiagnostix-Veterinary Pathology Services, 257 Boshoff Street, Pietermaritzburg, South Africa. ${ }^{5}$ College of Agriculture and Environmental Sciences, University of South Africa, Christiaan De Wet/Pioneer Dr., Florida, South Africa.

\section{Acknowledgements}

We would like to thank the Anthrax Advisory Committee.

\section{Competing interests}

The authors declare that they have no competing interests.

\section{Availability of data and materials}

All data generated or analysed during this study are included in this published article.

\section{Consent for publication}

Not applicable.

\section{Ethics approval and consent to participate}

Not applicable.

\section{Funding}

This study was funded by the National Research Foundation (NRF) of South Africa.

\section{Publisher's Note}

Springer Nature remains neutral with regard to jurisdictional claims in published maps and institutional affiliations.

Received: 20 March 2018 Accepted: 20 April 2018

Published online: 02 May 2018

\section{References}

1. Farrar WE. Anthrax: from mesopotamia to molecular biology. Pharos Alpha Omega Alpha Honor Med Soc. 1995;58(2):35-8.

2. Hugh-Jones ME, de Vos V. Anthrax and wildlife. Rev Sci Tech. 2002;21(2):359-83.

3. Organization WH. Epizootics IOo: anthrax in humans and animals Geneva: World Health Organization; 2008.

4. Leppla SH. Anthrax toxin edema factor: a bacterial adenylate cyclase that increases cyclic AMP concentrations of eukaryotic cells. Proc Natl Acad Sci USA. 1982;79(10):3162-6.

5. Sterne M. Variation in Bacillus anthracis. Onderstepoort J Vet Sci Anim Ind. 1937:8:271-349.

6. Koehler TM. Bacillus anthracis physiology and genetics. Mol Aspects Med. 2009:30(6):386-96.

7. Sterne M. The effects of different carbon dioxide concentrations on the growth of virulent anthrax strains: pathogenicity and immunity tests on Guineapigs and sheep with anthrax variants derived from virulent strains. Onderstepoort J Vet Sci. 1937;9:49-67.

8. Okinaka R, Cloud K, Hampton O, Hoffmaster A, Hill K, Keim P, Koehler T, Lamke G, Kumano S, Manter D, et al. Sequence, assembly and analysis of pX01 and pX02. J Appl Microbiol. 1999;87(2):261-2.

9. Pannucci J, Okinaka RT, Sabin R, Kuske CR. Bacillus anthracis pXO1 plasmid sequence conservation among closely related bacterial species. J Bacteriol. 2002;184(1):134-41.

10. Mock M, Fouet A. Anthrax. Annu Rev Microbiol. 2001;55:647-71.

11. Mock M, Mignot T. Anthrax toxins and the host: a story of intimacy. Cell Microbiol. 2003;5(1):15-23.

12. Green BD, Battisti L, Koehler TM, Thorne CB, Ivins BE. Demonstration of a capsule plasmid in Bacillus anthracis. Infect Immun. 1985;49(2):291-7.

13. Drysdale M, Bourgogne A, Hilsenbeck SG, Koehler TM. atXA controls Bacillus anthracis capsule synthesis via acpA and a newly discovered regulator, acpB. J Bacteriol. 2004;186(2):307-15.

14. Viljoen PR. Anthrax in South Africa. In: 13th and 14th reports of the director of veterinary education and research, Onderstepoort, South Africa; 1928.

15. Sterne M. Distribution and economic importance of anthrax. Fed Proc. 1967;26(5):1493-5.

16. Pienaar UD. Epidemiology of anthrax in wild animals and the control of anthrax epizootics in the Kruger National Park, South Africa. Fed Proc. 1967;26(5):1496-502.

17. Smith KL, DeVos V, Bryden H, Price LB, Hugh-Jones ME, Keim P. Bacillus anthracis diversity in Kruger National Park. J Clin Microbiol. 2000;38(10):3780-4.

18. De Vos V, Bryden H. Anthrax in the Kruger National Park: temporal and spatial patterns of disease occurrence. Salisbury Med Bull. 1996;87:26-31.

19. Odendaal MW, Pieterson PM, de Vos V, Botha AD. The antibiotic sensitivity patterns of Bacillus anthracis isolated from the Kruger National Park. Onderstepoort J Vet Res. 1991;58(1):17-9.

20. De Vos V. The ecology of anthrax in the Kruger National Park, South Africa. Salisbury Med Bull Suppl. 1990;68:19-23.

21. Van Ert MN, Easterday WR, Huynh LY, Okinaka RT, Hugh-Jones ME, Ravel J, Zanecki SR, Pearson T, Simonson TS, U'Ren JM, et al. Global genetic population structure of Bacillus anthracis. PLoS ONE. 2007;2(5):e461.

22. Good KM, Houser A, Arntzen L, Turnbull PC. Naturally acquired anthrax antibodies in a cheetah (Acinonyx jubatus) in Botswana. J Wildl Dis. 2008;44(3):721-3.

23. Good KM, Marobela C, Houser AM. A report of anthrax in cheetahs (Acinonyx jubatus) in Botswana. J S Afr Vet Assoc. 2005;76(4):186.

24. Jager HG, Booker HH, Hubschle OJ. Anthrax in cheetahs (Acinonyx jubatus) in Namibia. J Wildl Dis. 1990:26(3):423-4.

25. Turnbull PC, Tindall BW, Coetzee JD, Conradie CM, Bull RL, Lindeque PM, Huebschle OJ. Vaccine-induced protection against anthrax in cheetah (Acinonyx jubatus) and black rhinoceros (Diceros bicornis). Vaccine. 2004:22(25-26):3340-7.

26. Durant SM. Predator avoidance, breeding experience and reproductive success in endangered cheetahs, Acinonyx jubatus. Anim Behav. 2000;60(1):121-30

27. Ellerbrok H, Nattermann H, Ozel M, Beutin L, Appel B, Pauli G. Rapid and sensitive identification of pathogenic and apathogenic Bacillus anthracis by real-time PCR. FEMS Microbiol Lett. 2002;214(1):51-9. 
28. Birdsell DN, Pearson T, Price EP, Hornstra HM, Nera RD, Stone N, Gruendike J, Kaufman EL, Pettus AH, Hurbon AN, et al. Melt analysis of mismatch amplification mutation assays (Melt-MAMA): a functional study of a cost-effective SNP genotyping assay in bacterial models. PLoS ONE. 2012;7(3):e32866.

29. Andrews S. FastQC: a quality control tool for high throughput sequence data. 2010. http://www.bioinformatics.babraham.ac.uk/projects/fastqc.

30. Bolger AM, Lohse M, Usadel B. Trimmomatic: a flexible trimmer for Illumina sequence data. Bioinformatics. 2014;30(15):2114-20.

31. Altschul SF, Gish W, Miller W, Myers EW, Lipman DJ. Basic local alignment search tool. J Mol Biol. 1990;215(3):403-10.

32. Darling AC, Mau B, Blattner FR, Perna NT. Mauve: multiple alignment of conserved genomic sequence with rearrangements. Genome Res. 2004; 14(7):1394-403.

33. Pruitt KD, Tatusova T, Klimke W, Maglott DR. NCBI reference sequences: current status, policy and new initiatives. Nucleic Acids Res. 2009;37((Database issue)):D32-6.

34. Li H, Durbin R. Fast and accurate short read alignment with BurrowsWheeler transform. Bioinformatics. 2009;25(14):1754-60.

35. Li H, Handsaker B, Wysoker A, Fennell T, Ruan J, Homer N, Marth G, Abecasis $G$, Durbin R. Genome project data processing $S$ : the sequence alignment/map format and SAMtools. Bioinformatics. 2009;25(16):2078-9.

36. McKenna A, Hanna M, Banks E, Sivachenko A, Cibulskis K, Kernytsky A, Garimella K, Altshuler D, Gabriel S, Daly M, et al. The genome analysis toolkit: a MapReduce framework for analyzing next-generation DNA sequencing data. Genome Res. 2010;20(9):1297-303.

37. Kumar S, Stecher G, Tamura K. MEGA7: molecular evolutionary genetics analysis version 7.0 for bigger datasets. Mol Biol Evol. 2016;33(7):1870-4.

38. Butler J, MacCallum I, Kleber M, Shlyakhter IA, Belmonte MK, Lander ES, Nusbaum C, Jaffe DB. ALLPATHS: de novo assembly of whole-genome shotgun microreads. Genome Res. 2008;18(5):810-20.

39. Pearson T, Okinaka RT, Foster JT, Keim P. Phylogenetic understanding of clonal populations in an era of whole genome sequencing. Infect Genet Evol. 2009;9(5):1010-9.

40. Vergnaud G, Girault G, Thierry S, Pourcel C, Madani N, Blouin Y. Comparison of French and Worldwide Bacillus anthracis strains favors a recent, post-Columbian origin of the predominant North-American Clade. PLoS ONE. 2016;11(2):e0146216.

41. Derzelle S, Girault G, Kokotovic B, Angen O. Whole genome-sequencing and phylogenetic analysis of a historical collection of bacillus anthracis strains from Danish cattle. PLOS ONE. 2015;10(8):e0134699.

42. Girault G, Blouin Y, Vergnaud G, Derzelle S. High-throughput sequencing of Bacillus anthracis in France: investigating genome diversity and population structure using whole-genome SNP discovery. BMC Genomics. 2014;15:288.
Ready to submit your research? Choose BMC and benefit from:

- fast, convenient online submission

- thorough peer review by experienced researchers in your field

- rapid publication on acceptance

- support for research data, including large and complex data types

- gold Open Access which fosters wider collaboration and increased citations

- maximum visibility for your research: over 100M website views per year

At BMC, research is always in progress.

Learn more biomedcentral.com/submissions 